\title{
A Novel Method of Robust Trajectory Linearization Control Based on Disturbance Rejection
}

\author{
Xingling Shao ${ }^{1,2}$ and Honglun Wang ${ }^{1,2}$ \\ ${ }^{1}$ Unmanned Aerial Vehicle Research Institute, Beijing University of Aeronautics and Astronautics, Beijing 100191, China \\ ${ }^{2}$ Science and Technology on Aircraft Control Laboratory, Beijing University of Aeronautics and Astronautics, Beijing 100191, China \\ Correspondence should be addressed to Honglun Wang; hl_wang_2002@126.com
}

Received 30 December 2013; Revised 17 March 2014; Accepted 28 March 2014; Published 24 April 2014

Academic Editor: ShengJun Wen

Copyright (c) $2014 \mathrm{X}$. Shao and H. Wang. This is an open access article distributed under the Creative Commons Attribution License, which permits unrestricted use, distribution, and reproduction in any medium, provided the original work is properly cited.

\begin{abstract}
A novel method of robust trajectory linearization control for a class of nonlinear systems with uncertainties based on disturbance rejection is proposed. Firstly, on the basis of trajectory linearization control (TLC) method, a feedback linearization based control law is designed to transform the original tracking error dynamics to the canonical integral-chain form. To address the issue of reducing the influence made by uncertainties, with tracking error as input, linear extended state observer (LESO) is constructed to estimate the tracking error vector, as well as the uncertainties in an integrated manner. Meanwhile, the boundedness of the estimated error is investigated by theoretical analysis. In addition, decoupled controller (which has the characteristic of well-tuning and simple form) based on LESO is synthesized to realize the output tracking for closed-loop system. The closed-loop stability of the system under the proposed LESO-based control structure is established. Also, simulation results are presented to illustrate the effectiveness of the control strategy.
\end{abstract}

\section{Introduction}

Trajectory linearization control (TLC) is a novel nonlinear tracking and decoupling control method, which combines an open-loop nonlinear dynamic inversion and a linear time-varying (LTV) feedback stabilization, which guarantees that TLC's output achieves exponential stability along the nominal trajectory. Therefore, owing to the specific structure, it provides a certain extent of robust stability and can be capable of rejecting disturbance in nature, for which TLC has been successfully applied to missile and reusable launch vehicle flight control systems [1,2] and tripropeller UAV [3], helicopter [4], and fixed-wing vehicle [5].

However, in [6], theoretical analysis based on singular perturbation is proposed, which demonstrates that TLC can achieve local exponential stability because only linear term for original nonlinear system is ultimately reserved. In other words, when external and internal uncertainties are large enough to surpass the stability domain provided by TLC, the performance of the system will degrade significantly.
Thus, with the consideration of limitations of TLC in presence of uncertainties, how to enhance or improve the robustness and performance of TLC is becoming one of the active topics in control community recently [4, 7-14]. So far, the existing approach adopted by researchers can be classified as follows. By employing the excellent ability of neutral network $[4,8-11]$ or fuzzy logic $[12,13,15,16]$ in approximating the nonlinear functions, the unknown disturbances and uncertainties can be estimated and cancelled in enhanced control law, and thus the nominal performance of system can be recovered. Therefore, main research works are focused on the following aspects: (i) the construction of neutral network structure and fuzzy logic rules and (ii) the stability discussion of the compound system based on the estimated uncertainties. For instance, in [9], an adaptive neural network technique for nonlinear systems based on TLC is firstly proposed. The robustness and the stability of the proposed control scheme are also analyzed. A similar type of adaptive neural network TLC algorithm is also proposed through single hidden layer neutral networks (SHLNN) and radical basis function 
(RBF) neural network in [9-11]. In [12, 15, 16], TakagiSugeno (T-S) fuzzy system is applied to approximate the unknown functions in the system. Based on $[12,13]$ proposed a robust adaptive TLC(RATLC) algorithm, wherein only one parameter needs to be adapted on line, but there are too many design parameters to be chosen. Unlike the methods mentioned above, in [14], by using PD-eigenvalue assignment method, trajectory linearization observer is designed to cancel the uncertainties, but the design process seems cumbersome and the results are not satisfactory. Among the literatures mentioned above, one limitation which must be taken into account is that due to the complexity of the theory, it is overwhelmingly difficult to provide a guideline to tune the corresponding parameters, especially those which will influence the system performance greatly. In addition, the construction of fuzzy rules in T-S system usually needs certain extent of expertise knowledge. The drawbacks mentioned above will unavoidably increase the complexity of design procedure in engineering practice.

It is not difficult to recognize that the focal point of [7-14] is how to extract and estimate external disturbance and unknown dynamics by the known knowledge. In fact, there are many observers characterized in terms of state space formulation, as shown in [17], including the unknown input observer (UIO), the disturbance observer (DOB), and the extended state observer (ESO) which includes nonlinear ESO (NESO) and linear ESO (LESO) (when the structure of observer is chosen in nonlinear form, it refers to the term NESO, otherwise the term LESO). UIO is one of the earliest disturbances estimators, where the external disturbance is formulated as an augmented state and estimated using a state observer. Similar to UIO, ESO is also a state space approach. What sets ESO apart from UIO and DOB is that it is conceived to estimate not only the external disturbance but also plant dynamics. Furthermore, ESO requires the least amount of plant information. To be specific, only the relative order of system should be known. It is worth pointing out that, compared with NESO, LESO is greatly simplified with a single tuning parameter, that is, the bandwidth of LESO. Due to the excellent capability of LESO in estimating the unknown uncertainties, there have been many successful applications including biomechanics [18] and multivariable jet engines [19].

Above all, the essence of this problem is really disturbance rejection, with the notion of disturbance generalized to symbolize the uncertainties, both internal and external to the plant [20]. Central to this novel design framework proposed is the ability of LESO to estimate both the internal dynamics and external disturbances of the plant in real time. The major contributions of this paper are as follows.

(i) This is the first paper that employs LESO to improve the robustness and capability in disturbance rejection for TLC. Compared with methods proposed in [914], the novel controller can achieve fast and accurate response via effective compensation for unmodeled error and disturbances.

(ii) Unlike the conclusions on stability made by [9-14], the stability analysis in this paper not only gives the statement about the convergence of tracking error but also provides a viable guideline to select the parameters of controller; hence the complicate selection of $\mathrm{PD}$-eigenvalues via $\mathrm{PD}$-spectrum theorem which is widely used in [9-14] as a typical method can be avoided.

(iii) Compared with [9-14], only two parameters of the proposed method need to be tuned, which makes it extremely simple and practical to implement in real practice.

The paper is organized as follows. The review of TLC and controller design procedure based on LESO are presented in Sections 2 and 3, respectively. In Section 4, the analysis of closed-loop system error dynamics is given. Simulation results and discussion are shown in Section 5. The paper ends with a few concluding remarks in Section 6.

\section{Review of TLC}

Consider a multi-input multi-output (MIMO) nonlinear system:

$$
\begin{aligned}
\dot{\mathbf{x}} & =\mathbf{f}(\mathbf{x})+\mathbf{g}_{1}(\mathbf{x}) u+\mathbf{g}_{2}(\mathbf{x}) \mathbf{d}(\mathbf{x}), \\
\mathbf{y} & =\mathbf{h}(\mathbf{x}),
\end{aligned}
$$

where $\mathbf{x} \in \mathbf{R}^{n}$, $\mathbf{u} \in \mathbf{R}^{m}$, and $\mathbf{y} \in \mathbf{R}^{p}$ represent the state, the control input, and the output of the system, respectively. $\mathbf{f}(\mathbf{x})$, $\mathbf{g}_{\mathbf{1}}(\mathbf{x}), \mathbf{g}_{2}(\mathbf{x})$, and $\mathbf{h}(\mathbf{x})$ are smooth and bounded nonlinear function with appropriate dimensions. And $\mathbf{d}(\mathbf{x}) \in \mathbf{R}^{n}$ represents the unknown modeling error and external disturbance. Besides, $\mathbf{g}_{\mathbf{1}}(\mathbf{x})$ and $\mathbf{g}_{\mathbf{2}}(\mathbf{x})$ satisfy the matching conditions; namely, there exists a nonlinear matrix $\mathbf{g}_{0}(\mathbf{x}) \in R^{n \times m}$ such that

$$
\mathbf{g}_{0}(\mathbf{x}) \mathbf{g}_{1}(\mathbf{x})=\mathbf{g}_{2}(\mathbf{x}) .
$$

Firstly, without consideration of disturbance described by $\mathbf{d}(\mathbf{x})$, according to the design process of TLC method, the nominal control $\overline{\mathbf{u}}$, the nominal state $\overline{\mathbf{x}}$, and the nominal output $\overline{\mathbf{y}}$ will satisfy the following system:

$$
\begin{aligned}
& \dot{\overline{\mathbf{x}}}=\mathbf{f}(\overline{\mathbf{x}})+\mathbf{g}_{\mathbf{1}}(\overline{\mathbf{x}}) \overline{\mathbf{u}}, \\
& \overline{\mathbf{y}}=\mathbf{h}(\overline{\mathbf{x}}) .
\end{aligned}
$$

Let $=\overline{\mathbf{x}}+\mathbf{e}$ and $\mathbf{u}=\overline{\mathbf{u}}+\widetilde{\mathbf{u}}$; the tracking error dynamics can be described as

$$
\begin{aligned}
\dot{\mathbf{e}}= & \mathbf{f}(\overline{\mathbf{x}}+\mathbf{e})+\mathbf{g}_{1}(\overline{\mathbf{x}}+\mathbf{e})(\overline{\mathbf{u}}+\widetilde{\mathbf{u}})+\mathbf{g}_{2}(\mathbf{x}) \mathbf{d} \\
& -\mathbf{f}(\overline{\mathbf{x}})-\mathbf{g}_{1}(\overline{\mathbf{x}}) \bar{u}=\mathbf{F}(\overline{\mathbf{x}}, \overline{\mathbf{u}}, \mathbf{e}, \widetilde{\mathbf{u}})+\mathbf{g}_{2}(\mathbf{x}) \mathbf{d} .
\end{aligned}
$$

Since $\overline{\mathbf{x}}, \overline{\mathbf{u}}$ in (4) can be viewed as the time-varying parameters of the system, (4) can be simply written as

$$
\dot{\mathbf{e}}=\mathbf{F}(\overline{\mathbf{x}}, \overline{\mathbf{u}}, \mathbf{e}, \widetilde{\mathbf{u}})+\mathbf{g}_{2}(\mathbf{x}) \mathbf{d}=\mathbf{F}(t, \mathbf{e})+\mathbf{g}_{2}(\mathbf{x}) \mathbf{d} .
$$

Consider the LTV system derived by Taylor expansion at the equilibrium point $(\overline{\mathbf{x}}, \overline{\mathbf{u}})$ for (5); we have

$$
\dot{\mathbf{e}}=\mathbf{A}(\mathbf{t}) \mathbf{e}+\mathbf{B}(\mathbf{t}) \widetilde{\mathbf{u}}+\mathbf{g}_{2}(\mathbf{x}) \mathbf{d},
$$

where $\mathbf{A}(\mathbf{t})=\left.\left(\partial \mathbf{f} / \partial \mathbf{x}+\left(\partial \mathbf{g}_{1} / \partial \mathbf{x}\right) \mathbf{u}\right)\right|_{(\overline{\mathbf{x}}, \overline{\mathbf{u}})}$ and $\mathbf{B}(\mathbf{t})=\left.\mathbf{g}_{\mathbf{1}}(\mathbf{x})\right|_{(\overline{\mathbf{x}}, \overline{\mathbf{u}})}$. 
Assume that systems (5) and (6) satisfy the assumptions stated as follows.

Assumption 1. Let $\mathbf{e}=0$ be an isolated equilibrium point for (5) when $\mathbf{d}=0$, where $\mathbf{F}:[0, \infty) \times D_{e} \rightarrow R^{n}$ is continuously differentiable, $D_{e}=\left\{\mathbf{e} \in R^{n} \mid\|\mathbf{e}\|<R_{e}\right\}$, and the Jacobian matrix $[\partial \mathbf{F} / \partial t]$ is bounded and Lipschitz on $D_{e}$, uniformly in $t$.

Assumption 2. The system matrices pair $(\mathbf{A}(\mathbf{t}), \mathbf{B}(\mathbf{t}))$ in $(6)$ is uniformly completely controllable.

According to Assumption 2, we can design an LTV feedback control law $\widetilde{\mathbf{u}}=K(t) \mathbf{e}$ for the LTV system (6) when $\mathbf{d}=0$, the solution of system (6) can converge to zero exponentially. For simplicity, let $A_{c}(t)=A(t)+B(t) K(t)$, where $A_{c}(t)$ is Hurwitz. The parameters in $A_{c}(t)$ can be chosen by using $\mathrm{PD}$-spectrum theorem. The detailed design process of the nominal controller $\overline{\mathbf{u}}$ and the LTV feedback controller $\widetilde{\mathbf{u}}$ can be found in [1-3].

\section{Controller Design Based on LESO}

With the consideration of control quality for closed-loop system, the augmented tracking error in forms of PI can be written in the following state space form:

$$
\begin{aligned}
& \dot{\mathbf{e}}_{\mathrm{I}}=\mathbf{e}_{\mathrm{P}}, \\
& \dot{\mathbf{e}}_{\mathrm{p}}=\mathbf{A}(\mathbf{t}) \mathbf{e}_{\mathrm{p}}+\mathbf{B}(\mathbf{t}) \widetilde{\mathbf{u}}+\mathbf{g}_{2}(\mathbf{x}) \mathbf{d} .
\end{aligned}
$$

Assumption 3. The state vector $\mathbf{e}_{\mathbf{I}}$ in (7) is measurable.

Let $\xi=\left[\mathbf{e}_{\mathbf{I}}, \mathbf{e}_{\mathbf{P}}\right]^{T}=\left[\mathbf{e}_{1, I}, \mathbf{e}_{2, I}, \ldots, \mathbf{e}_{n, I}, \mathbf{e}_{1, P}, \mathbf{e}_{2, P}, \ldots, \mathbf{e}_{n, P}\right]^{T}$, and define $\mathbf{e}_{\mathbf{I}}$ as the output of new LTV system composed of (7) and (8); then the tracking error dynamics can be rewritten as

$$
\begin{aligned}
& \dot{\xi}=\left[\begin{array}{ll}
\mathbf{0}_{n \times n} & \mathbf{I}_{n \times n} \\
\mathbf{0}_{n \times n} & \mathbf{A}(\mathbf{t})
\end{array}\right] \xi+\left[\begin{array}{l}
\mathbf{0}_{n \times n} \\
\mathbf{B}(\mathbf{t})
\end{array}\right] \widetilde{\mathbf{u}}+\left[\begin{array}{c}
\mathbf{0}_{n \times n} \\
g_{2}(x)
\end{array}\right] \mathbf{d}, \\
& \mathbf{y}=\left[\begin{array}{ll}
\mathbf{I}_{1 \times n} & \mathbf{0}_{1 \times n}
\end{array}\right]\left[\begin{array}{l}
\mathbf{e}_{\mathbf{I}} \\
\mathbf{e}_{\mathbf{P}}
\end{array}\right] .
\end{aligned}
$$

It is obvious that, with the relative order and system order of (9) being $2 n$, the problem on the zero-dynamics subsystem does not exist.

Meanwhile, define

$$
\begin{aligned}
& {\left[\begin{array}{c}
F_{1}(\overline{\mathbf{x}}, \overline{\mathbf{u}}, t) \\
F_{2}(\overline{\mathbf{x}}, \overline{\mathbf{u}}, t) \\
\vdots \\
F_{n}(\overline{\mathbf{x}}, \overline{\mathbf{u}}, t)
\end{array}\right]} \\
& =\left[\begin{array}{cccc}
a_{11}(\overline{\mathbf{x}}, \overline{\mathbf{u}}, t) & a_{12}(\overline{\mathbf{x}}, \overline{\mathbf{u}}, t) & \cdots & a_{1 n}(\overline{\mathbf{x}}, \overline{\mathbf{u}}, t) \\
a_{21}(\overline{\mathbf{x}}, \overline{\mathbf{u}}, t) & a_{22}(\overline{\mathbf{x}}, \overline{\mathbf{u}}, t) & \cdots & a_{2 n}(\overline{\mathbf{x}}, \overline{\mathbf{u}}, t) \\
\vdots & \vdots & \vdots & \vdots \\
a_{n 1}(\overline{\mathbf{x}}, \overline{\mathbf{u}}, t) & a_{n 2}(\overline{\mathbf{x}}, \overline{\mathbf{u}}, t) & \cdots & a_{n n}(\overline{\mathbf{x}}, \overline{\mathbf{u}}, t)
\end{array}\right] \\
& \quad \times\left[\begin{array}{c}
e_{1, p} \\
e_{2, p} \\
\vdots \\
e_{n, p}
\end{array}\right],
\end{aligned}
$$

$$
\left[\begin{array}{c}
\widetilde{U}_{1} \\
\widetilde{U}_{2} \\
\vdots \\
\widetilde{U}_{n}
\end{array}\right]=\left[\begin{array}{cccc}
b_{11}(\overline{\mathbf{x}}, t) & b_{12}(\overline{\mathbf{x}}, t) & \cdots & b_{1 m}(\overline{\mathbf{x}}, t) \\
b_{21}(\overline{\mathbf{x}}, t) & b_{21}(\overline{\mathbf{x}}, t) & \cdots & b_{2 m}(\overline{\mathbf{x}}, t) \\
\vdots & \vdots & \vdots & \vdots \\
b_{n 1}(\overline{\mathbf{x}}, t) & b_{n 2}(\overline{\mathbf{x}}, t) & \cdots & b_{n m}(\overline{\mathbf{x}}, t)
\end{array}\right]\left[\begin{array}{c}
\widetilde{u}_{1} \\
\widetilde{u}_{2} \\
\vdots \\
\widetilde{u}_{m}
\end{array}\right],
$$

where $a_{i j}(\overline{\mathbf{x}}, \overline{\mathbf{u}}, t)$ represents the $i$ th row and the $j$ th column element of matrix $A(t)$ and $b_{i s}(\overline{\mathbf{x}}, t)$ represents the $i$ th row and the sth column element of matrix $B(t)$. In this case, the $i$ th tracking error subsystem can be formulated as

$$
\begin{array}{r}
\dot{e}_{i, I}=e_{i, P} \quad \dot{e}_{i, P}=F_{i}(\overline{\mathbf{x}}, \overline{\mathbf{u}}, t)+g_{2, i}(\mathbf{x}) \mathbf{d}+\widetilde{U}_{i}, \\
i \in \underline{n},
\end{array}
$$

where $g_{2, i}(\mathbf{x})$ represents the $i$ th row element of $\mathbf{g}_{2}(\mathbf{x})$, by introducing virtual control variable $v_{i}$, which takes the form of

$$
v_{i}=F_{i}(\overline{\mathbf{x}}, \overline{\mathbf{u}}, t)+g_{2, i}(\mathbf{x}) \mathbf{d}+\widetilde{U}_{i} .
$$

For subsystem (11), if the uncertainties in (12) are known, then the controller can be designed by feedback linearization method as

$$
\widetilde{U}_{i}=-k_{1, i} e_{i, P}-k_{2, i} e_{i, I}-g_{2, i}(\mathbf{x}) \mathbf{d}-F_{i}(\overline{\mathbf{x}}, \overline{\mathbf{u}}, t) .
$$

However, the control law cannot be synthesized unless $\mathbf{d}$ is estimated by observers. To deal with the estimation issue in (13), LESO provides a novel frame to achieve the function of uncertainties.

For simplicity, let $e_{i, d}=F_{i}(\overline{\mathbf{x}}, \overline{\mathbf{u}}, t)+g_{2, i}(\mathbf{x}) \mathbf{d}$, which represent the lumped disturbance; assume that $e_{i, d}$ is differentiable and denote $\dot{e}_{i, d}=h_{i}(\mathbf{x}, \mathbf{d})$; then (11) can be written in an augmented state space form:

$$
\begin{aligned}
\dot{e}_{i, I} & =e_{i, P}, \\
\dot{e}_{i, P} & =e_{i, d}+\widetilde{U}_{i}=v_{i}, \\
\dot{e}_{i, d} & =h_{i}(\mathbf{x}, \mathbf{d}), \\
y_{i} & =e_{i, I}, \\
i & \in \underline{n} .
\end{aligned}
$$

So far, by adopting direct feedback linearization, the original tracking error dynamics which take the form of linear time-varying have been transformed to canonical integral-chain form. Consequently, for (14), since $e_{i, d}$ is now a state in the extended state model, LESO can be designed to estimate $e_{i, I}, e_{i, P}$, and $e_{i, d}$. With $\widetilde{U}_{i}$ and $e_{i, I}$ as inputs, a particular LESO of (14) is given as

$$
\begin{aligned}
& z_{i, 0}=z_{i, 1}-e_{i, I}, \\
& \dot{z}_{i, 1}=z_{i, 2}-l_{01} z_{i, 0}, \\
& \dot{z}_{i, 2}=z_{i, 3}+\widetilde{U}_{i}-l_{02} z_{i, 0}, \\
& \dot{z}_{i, 3}=-l_{03} z_{i, 0}+h_{i}(\mathbf{x}, \mathbf{d}), \\
& i \in \underline{n},
\end{aligned}
$$


where $l_{01}, l_{02}, l_{03}$ are the observer gain parameters to be chosen such that the characteristic polynomial $s^{3}+l_{01} s^{2}+$ $l_{02} s+l_{03}$ is Hurwitz. According to [21], let $s^{3}+l_{01} s^{2}+l_{02} s+$ $l_{03}=\left(s+w_{0}\right)^{3}$, where $w_{0}$ denotes the observer bandwidth, which becomes the only tuning parameter of the observer.

Remark 4. Although $\dot{e}_{i, d}=h_{i}(\mathbf{x}, \mathbf{d})$ can be assumed theoretically, in engineering practice, $e_{i, d}$ which contains information of unknown disturbances cannot be obtained in advance. So, in practice, we might as well set $h_{i}(\mathbf{x}, \mathbf{d})=0$ in (15).

Furthermore, define $E_{i}=\left[e_{i, I}, e_{i, P}, e_{i, d}\right]^{T}$ and its estimated states $Z_{i}=\left[z_{i, 1}, z_{i, 2}, z_{i, 3}\right]^{T}$; hence (14) and (15) can be rewritten in the following matrix form:

$$
\begin{aligned}
& \dot{E}_{i}=A E_{i}+B_{1} \widetilde{U}_{i}+B_{2} h_{i}(\mathbf{x}, \mathbf{d}), \\
& \dot{Z}_{i}=A Z_{i}+B_{1} \widetilde{U}_{i}+L\left(E_{i}-Z_{i}\right),
\end{aligned}
$$

where $A=\left[\begin{array}{lll}0 & 1 & 0 \\ 0 & 0 & 1 \\ 0 & 0 & 0\end{array}\right], B_{1}=\left[\begin{array}{lll}0 & 1 & 0\end{array}\right]^{T}, L=\left[\begin{array}{lll}l_{0} & 0 & 0 \\ l_{02} & 0 & 0 \\ l_{03} & 0 & 0\end{array}\right]$, and $B_{2}=\left[\begin{array}{lll}0 & 0 & 1\end{array}\right]^{T}$. Hence, estimated error of the observer can be directly calculated as

$$
\dot{E}_{i}-\dot{Z}_{i}=(A-L)\left(E_{i}-Z_{i}\right)+B_{2} h_{i}(\mathbf{x}, \mathbf{d}) .
$$

For simplicity, let $\widetilde{E}_{i o}=E_{i}-Z_{i}$ and $A_{1}=A-L$. Here, $A_{1}$ is Hurwitz for $l_{01}, l_{02}$, and $l_{03}$; then (17) can be reduced to

$$
\dot{\widetilde{E}}_{i o}=A_{1} \widetilde{E}_{i o}+B_{2} h_{i}(\mathbf{x}, \mathbf{d}) .
$$

Theorem 5. Assuming $h_{i}(\mathbf{x}, \mathbf{d})$ is bounded, there exists a positive constant $M_{1}$ such that $\left|h_{i}(\mathbf{x}, \mathbf{d})\right| \leq M_{1}$; then estimated errors of the observer described by (18) are bounded. Furthermore, estimated errors of the observer satisfy $\left\|\widetilde{E}_{i o}\right\| \leq M_{2}$ for $t \rightarrow \infty$, where $M_{2}>0$.

Proof. If there exist three different negative real eigenvalues for $A_{1}$, it follows that $-\lambda_{1}<-\lambda_{2}<-\lambda_{3}<0, \lambda_{i}>0(i=$ $1, \ldots, 3)$; thus there exists nonsingular matrix $T$, and one has

$$
A_{1}=T \operatorname{diag}\left\{-\lambda_{1},-\lambda_{2},-\lambda_{3}\right\} T^{-1} .
$$

Note that

$$
\begin{gathered}
\exp \left(A_{1} t\right)=T \operatorname{diag}\left\{-\exp \left(\lambda_{1} t\right),-\exp \left(\lambda_{2} t\right),\right. \\
\left.-\exp \left(\lambda_{3} t\right)\right\} T^{-1} .
\end{gathered}
$$

When $t>0$, let us choose $m_{\infty}$ norm for the matrix norm. It is obvious that $\left\|\exp \left(A_{1} t\right)\right\|_{m_{\infty}} \leq \beta \exp \left(-\lambda_{1} t\right)(t>0)$, where $\beta>0$. The response of (18) can be written as

$$
\begin{array}{r}
\widetilde{E}_{\text {io }}(t)=\exp \left(A_{1} t\right) \widetilde{E}_{i o}(0)+\int_{0}^{t} \exp \left(A_{1}(t-\tau)\right) B_{2} h_{i} d \tau \\
t>0 .
\end{array}
$$

Hence, we have

$$
\begin{aligned}
&\left\|\widetilde{E}_{i o}(t)\right\| \\
& \leq\left\|\exp \left(A_{1} t\right) \widetilde{E}_{i o}(0)\right\|+\left\|\int_{0}^{t} \exp \left(A_{1}(t-\tau)\right) B_{2} h_{i} d \tau\right\| \\
& \leq\left\|\exp \left(A_{1} t\right)\right\|_{m_{\infty}}\left\|\widetilde{E}_{i o}(0)\right\| \\
&+\int_{0}^{t}\left\|\exp \left(A_{1}(t-\tau)\right)\right\|_{m_{\infty}}\left\|B_{2}\right\|\left\|h_{i}\right\| d \tau \\
& \leq \beta\left\|\widetilde{E}_{i o}(0)\right\| \exp \left(-\lambda_{1} t\right) \\
&+\frac{M_{1} \beta}{\lambda_{1}}\left(1-\exp \left(-\lambda_{1} t\right)\right) \leq \frac{M_{1} \beta}{\lambda_{1}}=M_{2} .
\end{aligned}
$$

From Theorem 5, it can be concluded that the upper bound of the estimated error monotonously decreases with absolute value of dominant pole $\lambda_{1}$ of LESO, that is, the bandwidth. This viewpoint is similar with the conclusion derived in $[21,22]$.

With respect to $i$ th subsystem of LTV system, control law can be formulated as

$$
\widetilde{U}_{i}(t)=-z_{i, 3}+v_{i}\left(t, Z_{i}\right), \quad i \in \underline{n},
$$

where the term $v_{i}\left(t, Z_{i}\right)$ is responsible for rendering (14) with satisfactory control quality. We have the following.

Theorem 6. Suppose that the estimated errors of LESO satisfy $\lim _{t \rightarrow \infty}\left\|\widetilde{E}_{i o}\right\|_{2}=0$, with the control structure as (23); virtual control variable can be designed as $v_{i}\left(t, Z_{i}\right)=-k_{1} z_{i, 1}-k_{2} z_{i, 2}$, where $k_{1}, k_{2}$ are gain parameters to be chosen to make $s^{2}+$ $k_{2} s+k_{1}$ be Hurwitz. Thus, the LTV system composed by virtual control variable satisfies the following.

(1) The controller of the LTV system stated above satisfies

$$
\widetilde{\mathbf{u}}= \begin{cases}B^{-1}(t)\left[\widetilde{U}_{1}, \widetilde{U}_{2}, \ldots, \widetilde{U}_{n}\right]^{T} & n=m \\ B^{\dagger}(t)\left[\widetilde{U}_{1}, \widetilde{U}_{2}, \ldots, \widetilde{U}_{n}\right]^{T} & n \neq m,\end{cases}
$$

and furthermore, the LTV subsystems are decoupled with each other.

(2) $\lim _{t \rightarrow \infty}\|\mathbf{e}\|_{2}=0$.

Proof. With virtual control variable designed as $v_{i}\left(t, Z_{i}\right)=$ $-k_{1} z_{i, 1}-k_{2} z_{i, 2}$, substituting (23) into (14), the $i$ th subsystem can be written as

$$
\begin{aligned}
& \dot{e}_{i, I}=e_{i, P}, \\
& \dot{e}_{i, P}=e_{i, d}-z_{i, 3}-k_{1} z_{i, 1}-k_{2} z_{i, 2}, \quad i \in \underline{n} .
\end{aligned}
$$

$$
y_{i}=e_{i, I} .
$$



that

Note that $\lim _{t \rightarrow \infty}\left\|\widetilde{E}_{i o}\right\|_{2}=0$; it can be directly concluded $\lim _{t \rightarrow \infty} e_{i, I}=z_{i, 1}, \quad \lim _{t \rightarrow \infty} e_{i, p}=z_{i, 2}, \quad \lim _{t \rightarrow \infty} e_{i, d}=z_{i, 3}$.

Substituting (26) into (25), one has

$$
\begin{aligned}
& \dot{e}_{i, I}=e_{i, P}, \\
& \dot{e}_{i, P}=-k_{1} e_{i, I}-k_{2} e_{i, P}=v_{i}\left(t, Z_{i}\right), \\
& y_{i}=e_{i, I}, \\
& \quad i \in \underline{n} .
\end{aligned}
$$

It is obvious that the relationship between the output $y_{i}$ and virtual control variable $v_{i}\left(t, Z_{i}\right)$ of the $i$ th subsystem is single-input and single-output. That is to say, the LTV subsystems are decoupled with each other.

Here, without loss of generality, the gain parameters $k_{1}$, $k_{2}$ satisfy the following condition: $s^{2}+k_{2} s+k_{1}=\left(s+w_{c}\right)^{2}$, $w_{c}>0$. For the given $k_{1}, k_{2}$, the overall controller of LTV system can be calculated as

$$
\widetilde{\mathbf{u}}= \begin{cases}B^{-1}(t)\left[\widetilde{U}_{1}, \widetilde{U}_{2}, \ldots, \widetilde{U}_{n}\right]^{T} & n=m \\ B^{\dagger}(t)\left[\widetilde{U}_{1}, \widetilde{U}_{2}, \ldots, \widetilde{U}_{n}\right]^{T} & n \neq m,\end{cases}
$$

where $B^{\dagger}(t)$ denotes the generalized inverse of $B(t)$.

Next, we mainly prove the conclusion (2).

Let the tracking error of $i$ th subsystem be $E_{i}^{\prime}=$ $\left[\begin{array}{ll}e_{i, I} & e_{i, P}\end{array}\right]^{T}$; then the tracking error dynamics of $i$ th subsystem can be written as

$$
\dot{E}_{i}^{\prime}=A_{3} E_{i}^{\prime}+A_{4} \widetilde{E}_{i o}, \quad i \in \underline{n},
$$

where $A_{3}=\left[\begin{array}{cc}0 & 1 \\ -k_{1} & -k_{2}\end{array}\right], A_{4}=\left[\begin{array}{ccc}0 & 0 & 0 \\ -k_{1} & -k_{2} & -1\end{array}\right]$.

Since $\lim _{t \rightarrow \infty}\left\|\widetilde{E}_{i o}\right\|_{2}=0$, then for any $\phi>0$ there is a finite time $T_{1}>0$ such that $\left\|A_{4} \widetilde{E}_{i o}\right\| \leq \phi$ for all $t>T_{1}>0$. Then, the response of (29) can be written as

$$
E_{i}^{\prime}(t)=\exp \left(A_{3} t\right) E_{i}^{\prime}(0)+\int_{0}^{t} \exp \left(A_{3}(t-\tau)\right) A_{4} \widetilde{E}_{i o}(\tau) d \tau,
$$

When $t>T_{1}$, we have

$$
\begin{aligned}
\left\|E_{i}^{\prime}(t)\right\| \leq & \left\|\exp \left(A_{3} t\right) E_{i}^{\prime}(0)\right\| \\
& +\left\|\exp \left(A_{3} t\right)\right\|\left\|\int_{0}^{T_{1}} \exp \left(-A_{3} \tau\right) A_{4} \widetilde{E}_{i o}(\tau) d \tau\right\| \\
& +\int_{T_{1}}^{t}\left\|\exp \left(A_{3}(t-\tau)\right)\right\| \phi d \tau .
\end{aligned}
$$

Suppose that there exist two different real eigenvalues for $A_{3}$; it follows that $-\lambda_{1}^{\prime}<-\lambda_{2}^{\prime}<0, \lambda_{i}^{\prime}>0(i=1,2)$; thus there exists nonsingular matrix $T$, and one has

$$
A_{3}=T \operatorname{diag}\left\{-\lambda_{1}^{\prime},-\lambda_{2}^{\prime}\right\} T^{-1} .
$$

Similar to Theorem 5, $\left\|\exp \left(A_{3} t\right)\right\|_{m_{\infty}} \leq \beta_{1} \exp \left(-\lambda_{1}^{\prime} t\right)$, where $\beta_{1}>0$.

Hence, we have

$$
\begin{aligned}
\left\|E_{i}^{\prime}(t)\right\| \leq & \left\|\exp \left(A_{3} t\right) E_{i}^{\prime}(0)\right\| \\
& +\left\|\exp \left(A_{3} t\right)\right\|\left\|\int_{0}^{T_{1}} \exp \left(-A_{3} \tau\right) A_{4} \widetilde{E}_{i o}(\tau) d \tau\right\| \\
& +\phi\|T\|\left\|T^{-1}\right\| \beta_{1} \int_{T_{1}}^{t} \exp \left(-\lambda_{1}^{\prime}(t-\tau)\right) d \tau \\
= & \left\|\exp \left(A_{3} t\right) E_{i}^{\prime}(0)\right\| \\
& +\left\|\exp \left(A_{3} t\right)\right\|\left\|\int_{0}^{T_{1}} \exp \left(-A_{3} \tau\right) A_{4} \widetilde{E}_{i o}(\tau) d \tau\right\| \\
& +\frac{\phi \beta_{1} \exp \left(-\lambda_{1}^{\prime}\left(t-T_{1}\right)\right)}{\left(-\lambda_{1}^{\prime}\right)}+\frac{\phi \beta_{1}}{\left(-\lambda_{1}^{\prime}\right)} .
\end{aligned}
$$

It can be seen that

$$
\lim _{t \rightarrow \infty}\left\|\exp \left(A_{3} t\right) E_{i}^{\prime}(0)\right\|=0,
$$

$$
\begin{gathered}
\lim _{t \rightarrow \infty}\left\|\exp \left(A_{3} t\right)\right\|\left\|\int_{0}^{T_{1}} \exp \left(-A_{3} \tau\right) A_{4} \widetilde{E}_{i o}(\tau) d \tau\right\|=0, \\
\frac{\lim _{t \rightarrow \infty} \phi \beta_{1} \exp \left(-\lambda_{1}^{\prime}\left(t-T_{1}\right)\right)}{\left(-\lambda_{1}^{\prime}\right)}=0 .
\end{gathered}
$$

Therefore, there exists $T_{2}>T_{1}>0$ such that

$$
\begin{array}{r}
\left\|\exp \left(A_{3} t\right) E_{i}^{\prime}(0)\right\| \leq \phi, \quad \forall t>T_{2}>0, \\
\left\|\exp \left(A_{3} t\right)\right\|\left\|\int_{0}^{T_{1}} \exp \left(-A_{3} \tau\right) A_{4} \widetilde{E}_{i o}(\tau) d \tau\right\| \leq \phi, \\
\forall t>T_{2}>0, \\
\frac{\phi \beta_{1} \exp \left(-\lambda_{1}^{\prime}\left(t-T_{1}\right)\right)}{\left(-\lambda_{1}^{\prime}\right)} \leq \phi, \quad \forall t>T_{2}>0 .
\end{array}
$$

Let $c^{\prime \prime}=\beta_{1} /\left(-\lambda_{1}^{\prime}\right)$; then we have $\left\|E_{i}^{\prime}(t)\right\| \leq\left(c^{\prime \prime}+3\right) \phi, \forall t>$ $T_{2}>0$.

Since $\phi$ can be arbitrarily small, it can be concluded that $\lim _{t \rightarrow \infty}\left\|E_{i}^{\prime}(t)\right\|=0, i \in \underline{n}$.

Since the LTV subsystems are decoupled with each other, the tracking errors of closed-loop system satisfy the following:

$$
\lim _{t \rightarrow \infty}\|\mathbf{e}\|_{2}=0 .
$$




\section{Stability Analysis of Closed-Loop System}

It is worth pointing out that conclusion (2) of Theorem 6 holds only if $\lim _{t \rightarrow \infty}\left\|\widetilde{E}_{i o}\right\|_{2}=0$. Actually, according to Theorem 5, that is, $\left\|\widetilde{E}_{i o}\right\| \leq M_{2}$, the tracking error of $i$ th subsystem and the estimated error of LESO can be written in the following cascade structure:

$$
\begin{array}{r}
\dot{E}_{i}^{\prime}=A_{3} E_{i}^{\prime}+A_{4} \widetilde{E}_{i o}, \quad \dot{\widetilde{E}}_{i o}=A_{1} \widetilde{E}_{i o}+B_{2} h_{i}(\mathbf{x}, \mathbf{d}), \\
i \in \underline{n} .
\end{array}
$$

Theorem 7. For the tracking error dynamics described by (37), there exist gain parameters $k_{1}>0, k_{2}>0$, and positive constant $M_{3}>0$ such that $\left\|A_{4}\right\|_{2} \leq M_{3}$; then

$$
\lim _{t \rightarrow \infty}\left\|E_{i}^{\prime}\right\|_{2} \leq \frac{M_{1} M_{3} \beta \beta_{1}}{\lambda_{1} \lambda_{1}^{\prime}}, \quad i \in \underline{n},
$$

where $M_{1}, M_{3}, \beta$, and $\beta_{1}$ are constants related to the system dynamics and controller parameters and $-\lambda_{1},-\lambda_{1}^{\prime}\left(\lambda_{1}>0\right.$, $\left.\lambda_{1}^{\prime}>0\right)$ are dominant poles of LESO and controller, respectively.

Proof. From Theorem 6, the LTV subsystems are decoupled with each other. For simplicity, here, it is necessary to prove the ultimate tracking error bound of $i$ th subsystem. Conclusions obtained can be readily applied to the overall subsystems.

The solution of (37) can be written as

$$
E_{i}^{\prime}(t)=\exp \left(A_{3} t\right) E_{i}^{\prime}(0)+\int_{0}^{t} \exp \left(A_{3}(t-\tau)\right) A_{4} \widetilde{E}_{i o}(\tau) d \tau
$$

Similar to Theorem 6 , we have $\left\|\exp \left(A_{3} t\right)\right\|_{m_{\infty}} \leq \beta_{1}$ $\exp \left(-\lambda_{1}^{\prime} t\right)$, where $\beta_{1}>0$. From Theorem 5 , it follows that

$$
\begin{aligned}
\left\|\widetilde{E}_{i o}(\tau)\right\| \leq & \beta\left\|\widetilde{E}_{i o}(0)\right\| \exp \left(-\lambda_{1} \tau\right) \\
& +\frac{M_{1} \beta}{\lambda_{1}}\left(1-\exp \left(-\lambda_{1} \tau\right)\right) .
\end{aligned}
$$

Substituting the above inequality into (39), we can get

$$
\begin{aligned}
& \left\|E_{i}^{\prime}(t)\right\| \\
& \leq \beta_{1}\left\|E_{i}^{\prime}(0)\right\| \exp \left(-\lambda_{1}^{\prime} t\right) \\
& \quad+M_{3} \beta \beta_{1}\left\|\widetilde{E}_{i o}(0)\right\| \int_{0}^{t} \exp \left(-\lambda_{1}^{\prime}(t-\tau)\right) \exp \left(-\lambda_{1}^{\prime} \tau\right) d \tau \\
& \quad+\frac{M_{1} M_{3} \beta \beta_{1}}{\lambda_{1}} \int_{0}^{t} \exp \left(-\lambda_{1}^{\prime}(t-\tau)\right)\left(1-\exp \left(-\lambda_{1} \tau\right)\right) d \tau .
\end{aligned}
$$

It is usually desirable in observer design that $\lambda_{1}>\lambda_{1}^{\prime}>0$; that is, the observer dynamics are designed to be faster than the controller tracking error dynamics in order to recover the system performance by the singular perturbation theory. Thus, inequality (41) can be further expressed as

$$
\begin{aligned}
\left\|E_{i}^{\prime}(t)\right\| & \\
\leq & \beta_{1}\left\|E_{i}^{\prime}(0)\right\| \exp \left(-\lambda_{1}^{\prime} t\right) \\
& +\frac{M_{3} \beta \beta_{1}\left\|\widetilde{E}_{i o}(0)\right\|}{\lambda_{1}^{\prime}-\lambda_{1}}\left(\exp \left(-\lambda_{1} t\right)-\exp \left(-\lambda_{1}^{\prime} t\right)\right) \\
& +\frac{M_{1} M_{3} \beta \beta_{1}}{\lambda_{1} \lambda_{1}^{\prime}}\left(1-\exp \left(-\lambda_{1}^{\prime} t\right)\right) \\
& -\frac{M_{1} M_{3} \beta \beta_{1}}{\lambda_{1}\left(\lambda_{1}^{\prime}-\lambda_{1}\right)}\left(\exp \left(-\lambda_{1} t\right)-\exp \left(-\lambda_{1}^{\prime} t\right)\right) \\
\leq & \beta_{1}\left\|E_{i}^{\prime}(0)\right\| \exp \left(-\lambda_{1}^{\prime} t\right)+\frac{M_{3} \beta \beta_{1}\left\|\widetilde{E}_{i o}(0)\right\|}{\lambda_{1}-\lambda_{1}^{\prime}} \exp \left(-\lambda_{1}^{\prime} t\right) \\
& +\frac{M_{1} M_{3} \beta \beta_{1}}{\lambda_{1} \lambda_{1}^{\prime}}\left(1-\exp \left(-\lambda_{1}^{\prime} t\right)\right) \\
& -\frac{M_{1} M_{3} \beta \beta_{1}}{\lambda_{1}\left(\lambda_{1}-\lambda_{1}^{\prime}\right)} \exp \left(-\lambda_{1}^{\prime} t\right) .
\end{aligned}
$$

Let $L=\beta_{1}\left\|E_{i}^{\prime}(0)\right\|+M_{3} \beta \beta_{1}\left\|\widetilde{E}_{i o}(0)\right\| /\left(\lambda_{1}-\lambda_{1}^{\prime}\right)-M_{1} M_{3} \beta \beta_{1} /$ $\lambda_{1} \lambda_{1}^{\prime}-M_{1} M_{3} \beta \beta_{1} / \lambda_{1}\left(\lambda_{1}-\lambda_{1}^{\prime}\right)$ and the above inequality can be rearranged as

$$
\left\|E_{i}^{\prime}(t)\right\| \leq L \exp \left(-\lambda_{1}^{\prime} t\right)+\frac{M_{1} M_{3} \beta \beta_{1}}{\lambda_{1} \lambda_{1}^{\prime}}
$$

It can be seen that $\lim _{t \rightarrow \infty}\left\|E_{i}^{\prime}\right\|_{2} \leq M_{1} M_{3} \beta \beta_{1} / \lambda_{1} \lambda_{1}^{\prime}, i \in \underline{n}$.

From Theorem 7, the following conclusion can also be obtained: suppose that there exist positive constants $M_{1}$ and $M_{3}$ such that $\left\|A_{4}\right\|_{2} \leq M_{3},\left|h_{i}(\mathbf{x}, \mathbf{d})\right| \leq M_{1}$; then there exist LESO parameters and controller gain parameters $l_{01}>0$, $l_{02}>0, l_{03}>0, k_{1}>0, k_{2}>0$ such that the tracking errors of closed-loop system are bounded; that is, with respect to any bounded input, the output of closed-loop system is bounded; in other words, the closed-loop system is BIBO stable.

\section{Simulation Results and Discussion}

To demonstrate the effectiveness of the proposed approach, a numerical example is considered, which is described by Changsheng et al. [13]

$$
\begin{gathered}
\dot{\xi}=-\frac{\sin (4 \pi \xi)}{4 \pi \xi^{2}+1}+(2+\cos (7 \xi)) u, \\
y=\xi, \quad \xi(0)=0.5,
\end{gathered}
$$

where $u$ represents the input and $\xi$ represents the output. In fact, the affine nonlinear system described by (44) can 
represent a class of models existing widely in real practice, such as motor motion system.

According to the design procedure of the TLC method, the nominal input can be obtained:

$$
\bar{u}=\frac{1}{2+\cos (7 \bar{\xi})}\left[\dot{\bar{\xi}}+\frac{\sin (4 \pi \bar{\xi})}{4 \pi \bar{\xi}^{2}+1}\right] .
$$

To maintain causality, the derivative of $\bar{\xi}$ in (45) can be calculated through a pseudodifferentiator which takes the following form of transfer function:

$$
G(s)=\frac{5 s}{s+5}
$$

According to the design framework of TLC [1-3], a PI regulator can be designed by defining an augmented tracking error to improve the performance of the closed-loop system. The augmented tracking error is defined as follows:

$$
\mathbf{e}=\left[\begin{array}{l}
e_{I} \\
e_{P}
\end{array}\right]=\left[\begin{array}{c}
(\xi-\bar{\xi}) d t \\
\xi-\bar{\xi}
\end{array}\right]
$$
ten as

Correspondingly, the original system (44) can be rewrit-

$$
\begin{aligned}
& \dot{\mathbf{x}}=f(\mathbf{x})+g_{1}(\mathbf{x}) u, \\
& y=x_{2},
\end{aligned}
$$

where $\mathbf{x}=\left[\begin{array}{l}x_{1} \\ x_{2}\end{array}\right]=\left[\begin{array}{c}\int \xi d t \\ \xi\end{array}\right], f=\left[\begin{array}{c}x_{2} \\ -\sin \left(4 \pi x_{2}\right) /\left(4 \pi x_{2}^{2}+1\right)\end{array}\right], g_{1}=$ $\left[\begin{array}{c}0 \\ 2+\cos \left(7 x_{2}\right)\end{array}\right]$.

By linearizing (48) along the nominal trajectory $(\bar{x}, \bar{u})$, the time-varying matrices for the augmented error dynamics can be obtained:

$$
A(t)=\left[\begin{array}{cc}
0 & 1 \\
0 & a_{22}
\end{array}\right], \quad B(t)=\left[\begin{array}{l}
0 \\
b_{2}
\end{array}\right],
$$

where $a_{22}=-4 \pi \cos \left(4 \pi \bar{x}_{2}\right) /\left(4 \pi \bar{x}_{2}^{2}+1\right)+8 \pi \bar{x}_{2} \sin \left(4 \pi \bar{x}_{2}\right) /$ $\left(4 \pi \bar{x}_{2}^{2}+1\right)^{2}-7 \sin \left(7 \bar{x}_{2}\right) \bar{u}, b_{2}=2+\cos \left(7 \bar{x}_{2}\right)$.

The tracking and disturbance rejection performance of TLC combined with LESO are tested under the following different scenarios.

Case 1. There exist no unmodeled dynamics and disturbances.

Case 2. The unmodeled dynamics exist in the system described as

$$
d=1.5 \sin (2 \xi+1) .
$$

Case 3. Both unmodeled dynamics and external disturbances exist in the system described as

$$
d=1.5 \sin (2 \xi+1)+2 \sin (t+1) .
$$

Thus, the system (48) can be rewritten as

$$
\begin{aligned}
& \dot{\mathbf{x}}=f(\mathbf{x})+g_{1}(\mathbf{x}) u+g_{2}(\mathbf{x}) d, \\
& y=x_{2},
\end{aligned}
$$

where $g_{2}(\mathbf{x})=[0,1]^{T}$.

Suppose that the tracking error $\mathbf{e}_{\mathbf{I}}$ of LTV system is measurable, according to the method proposed; the controller of LTV system can be synthesized as follows:

$$
\widetilde{U}(t)=-z_{3}+v(t, Z)=-z_{3}-k_{1} z_{1}-k_{2} z_{2},
$$

where $Z=\left[z_{1}, z_{2}, z_{3}\right]^{T}$, which can be produced by the following dynamics:

$$
\begin{aligned}
& z_{0}=z_{1}-e_{I} . \\
& \dot{z}_{1}=z_{2}-l_{01} z_{0} \\
& \dot{z}_{2}=z_{3}+\widetilde{U}-l_{02} z_{0} \\
& \dot{z}_{3}=-l_{03} z_{0} .
\end{aligned}
$$

In this simulation, the tuning parameters are $w_{0}=$ $200 \mathrm{rad} / \mathrm{s}$ and $w_{c}=20 \mathrm{rad} / \mathrm{s}$. Correspondingly, $l_{01}=3 w_{o}$, $l_{02}=3 w_{o}^{2}, l_{01}=w_{o}^{3}, k_{1}=w_{c}^{2}$, and $k_{2}=2 w_{c}$.

Above all, the overall controller of the closed-loop system can be synthesized as follows:

$$
u=\bar{u}+\widetilde{u}=\bar{u}+B(t)^{\dagger} \widetilde{U}(t) .
$$

In order to compare conveniently, here, the control law of [13] is also given as follows:

$$
u=\bar{u}+\widetilde{u}=\bar{u}+K(t) \mathbf{e}-g_{0} v_{\mathrm{ad}},
$$

where $K(t)$ denotes gain matrix to be chosen by utilizing PDspectrum theorem of TLC, while $v_{\text {ad }}$ denotes the output of the robust adaptive controller constructed on the basis of T$S$ fuzzy system. The detailed design method can be found in [13]. Here, the design parameters to be chosen in [13] are outlined below, respectively, $Q(t)=12 I_{2}, \sigma=50, \gamma=5$, $\rho=0.5$, and $\lambda_{0}=0$.

Firstly, we suppose that the reference command is the same with [13], which can be described by

$$
\bar{y}=0.3 \sin \left(\frac{t}{2}\right)+0.5 \cos (t) \text {. }
$$

The tracking performance of original TLC method tested under the aforementioned scenarios is shown in Figure 1.

From the simulation in Figure 1, it can be observed that the output of closed-loop system can track the command closely in the absence of unmodeled dynamics or external disturbances. However, if there exist unmodeled dynamics or both unmodeled dynamics and external disturbances as stated in Figure 1, the tracking performance of TLC degrades remarkably. Thus, the original TLC method cannot meet the increasing demands on accuracy and robustness when larger disturbances are considered. 

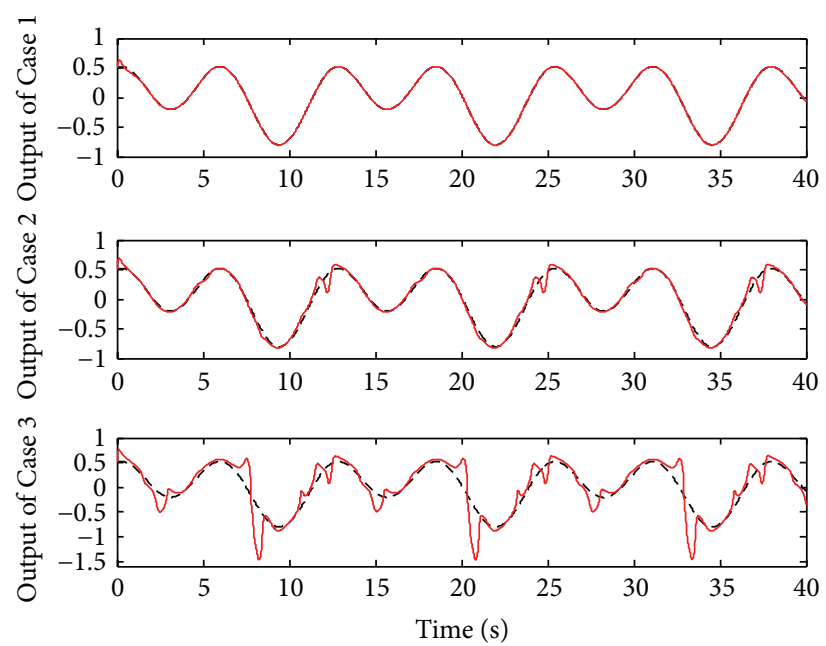

- - - Reference command

- Output response

FIGURE 1: Simulation results for original TLC method.
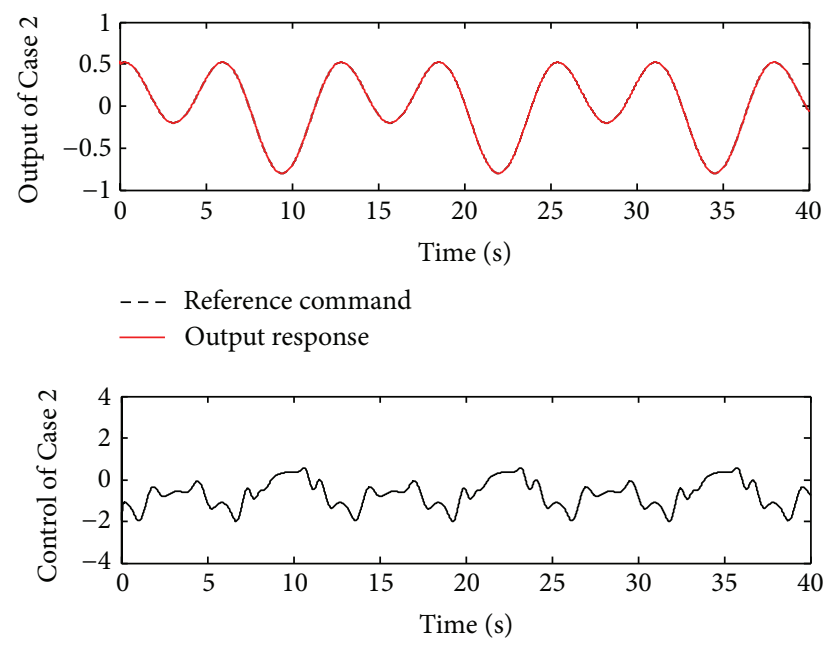

- Control input

FIGURE 2: Simulation results for proposed method under Case 2.

The performance for the proposed method and control scheme presented in [13] tested in the presence of the aforementioned uncertainties are shown in Figures 2-5, respectively. Meanwhile, in order to emphasize the advantage of the proposed method, tracking errors of the closed-loop system for the proposed method and the method in [13] are also illustrated in Figure 6, respectively.

From the simulation in Figures 2-6, it can be observed that the output of proposed method and the method in [13] can both track the command closely under aforementioned scenarios. Compared with Figures 4 and 5, Figures 2 and 3 clearly demonstrate that the proposed method has better performance in control quality such as tracking precision and robustness, especially in the presence of larger disturbances. Such performance can only be attributed to the ability of
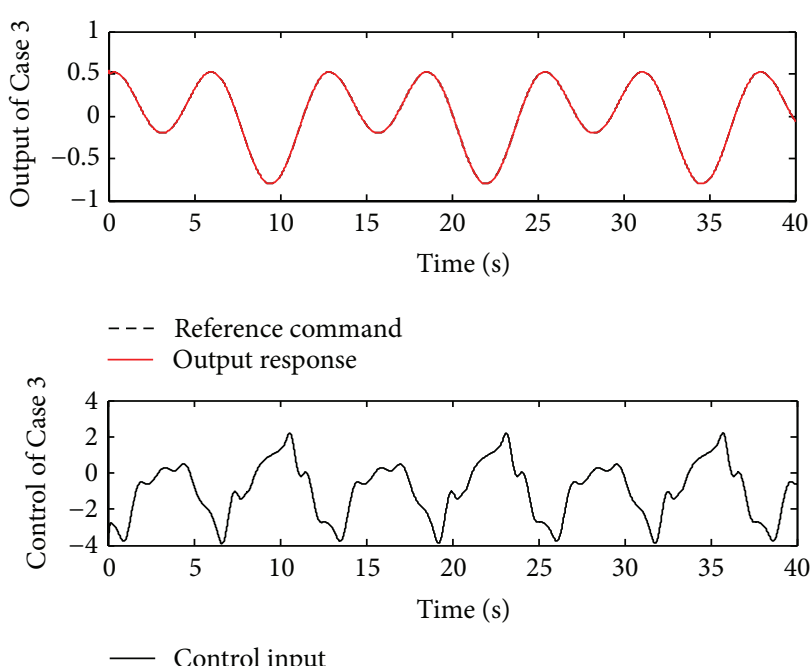

FIGURE 3: Simulation results for proposed method under Case 3.

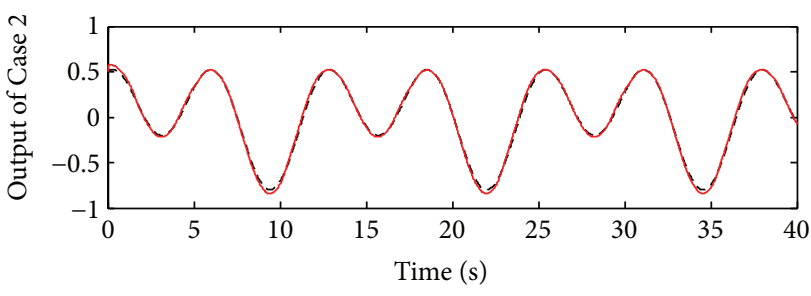

- - - Reference command

- Output response

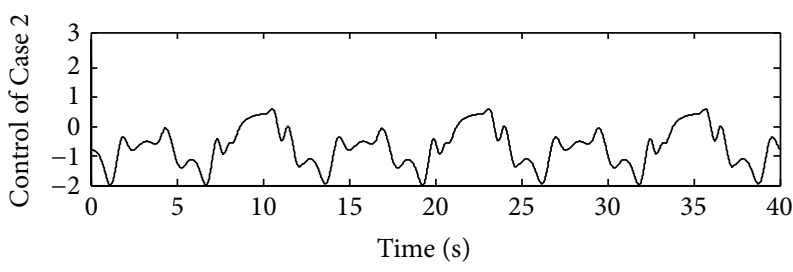

- Control input

FIgURE 4: Simulation results for the method in [13] under Case 2.

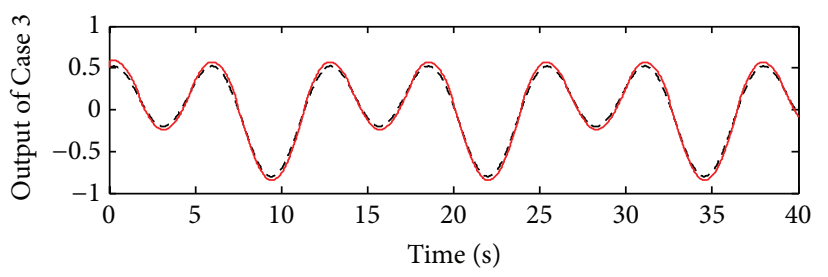

- - Reference output

- Output response

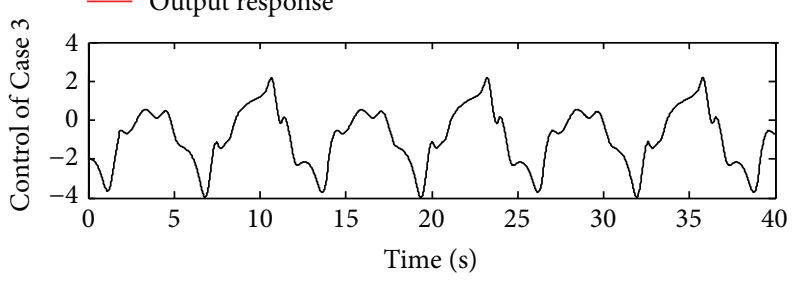

- Control input

FIGURE 5: Simulation results for the method in [13] under Case 3. 

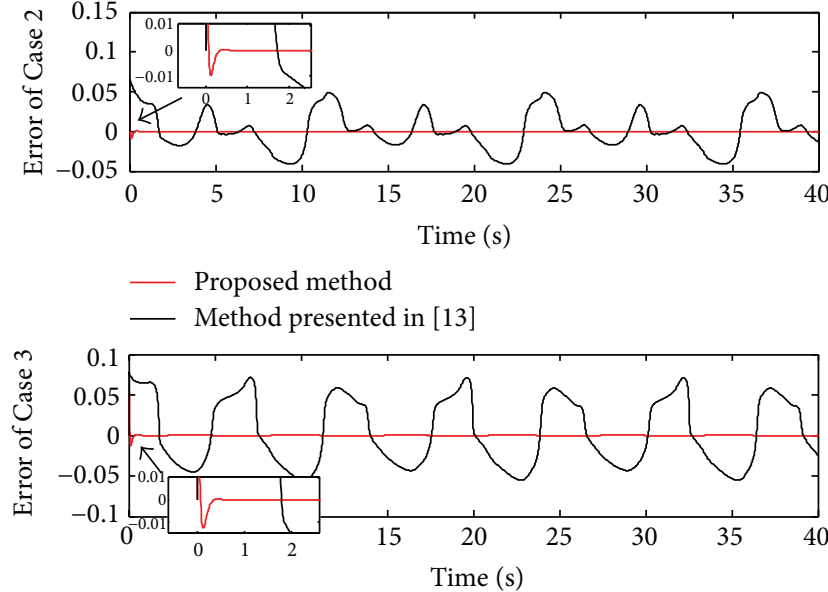

FIGURE 6: Tracking errors for proposed method and the method in [13].

LESO in obtaining an accurate estimation of the combined effect of unmodeled dynamics and external disturbances in real time. Moreover, the closed-loop tracking errors for the proposed method under Cases 2 and 3 all converge to zero quickly and ultimately maintain steadily in the neighborhood of zero. However, for the method proposed in [13], the upper bound of tracking error increases as more uncertainties are incorporated into lumped disturbances. Apparently, highly tracking accuracy for the method in [13] cannot be guaranteed in face of larger uncertainties.

To further demonstrate the relationship between the tracking error and the bandwidth, Figure 7 shows the simulation results using the reduced bandwidth $w_{0}=100 \mathrm{rad} / \mathrm{s}$ and $w_{c}=10 \mathrm{rad} / \mathrm{s}$. In addition, the curve for estimated error with different bandwidth of LESO is also given in Figure 8. The simulation results in Figure 7 obviously verify the validity of Theorems 6 and 7; that is, the ultimate upper bound of closed-loop tracking error monotonously decreases with the product of LESO's and controller's bandwidth. This conclusion provides a viable guideline to select the parameters of controller. Compared with the method in [13], the ultimate upper bound of tracking error can achieve the magnitude of $10^{-4}$. Moreover, Figure 8 shows that the upper bound of the estimated error for lumped disturbance decreases as bandwidth increases, which is coincided with Theorem 5 .

Next, in order to illustrate the control strategy can also work well when the desired trajectory proceeds with abrupt disturbance, we suppose a step disturbance with the amplitude of 3 at $t=15 \mathrm{~s}$ as the abrupt disturbance; in this case, control strategy proposed in [11] is considered to make comparison. The parameters of proposed method are kept unchanged, as mentioned previously. Figure 9 shows the tracking response for proposed method and the method in [11]. It is obvious that, compared with [11], the output of the proposed method tracks the reference command effectively in spite of abrupt disturbance at $t=15 \mathrm{~s}$. The tracking error can converge to a neighborhood of zero rapidly. However, for the method proposed in [11], the tracking error changes obviously when abrupt disturbance occurs. Thus, with LESO,

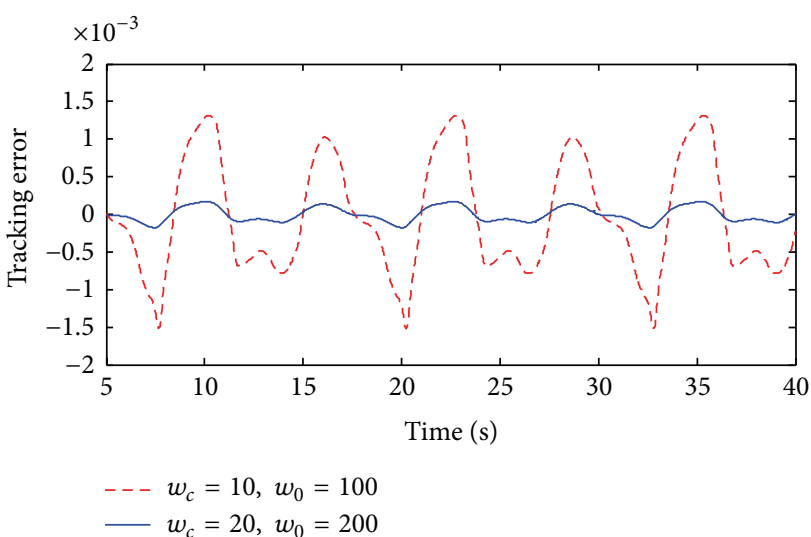

FIGURE 7: Tracking error for proposed method with different design parameters.

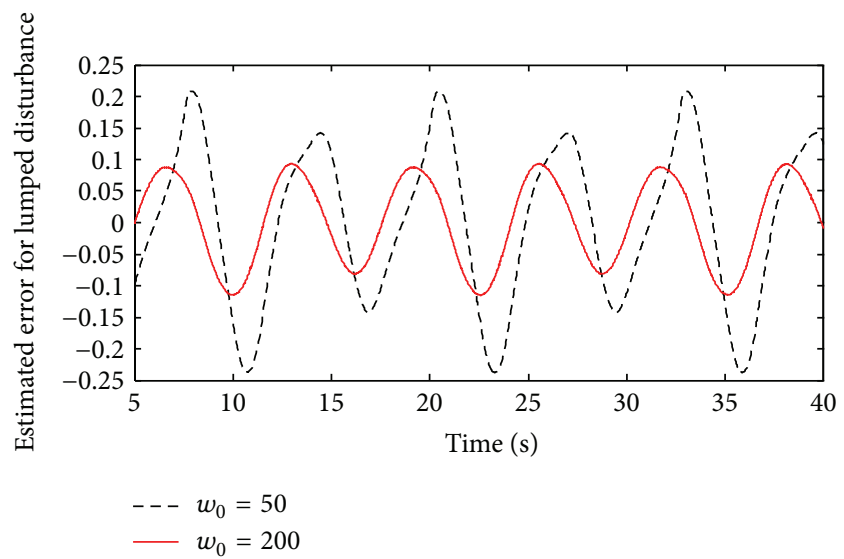

FIGURE 8: Estimated error for proposed method with different design parameters.

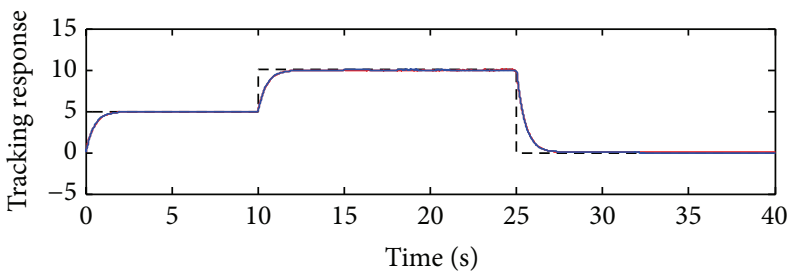

- - Reference command
— Proposed method
— Method presented in [13]

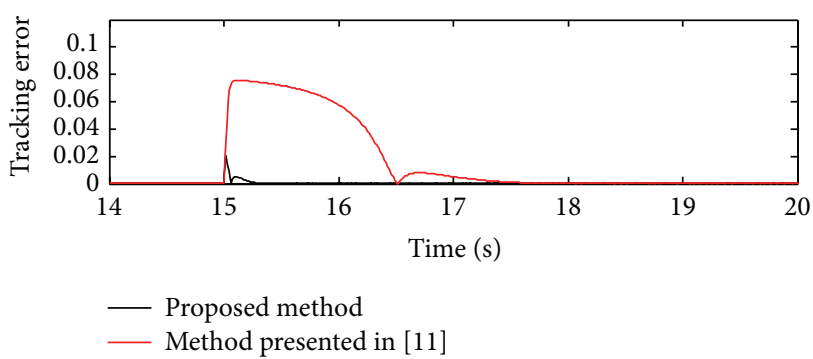

FIGURE 9: Tracking response for proposed method and the method in [11]. 
the capability of the proposed method in disturbance rejection is superior to that of the method proposed in [11].

Above all, compared with [9-14], only two parameters of the proposed method need to be tuned while maintaining the excellent performance such as disturbance rejection and tracking characteristics, which makes it extremely simple and practical. Both the stability analysis and the simulation study demonstrate the effectiveness and the robustness of the proposed method.

\section{Concluding Remarks}

The main result in this paper is the validation of proposed method through theoretical analysis and simulation. The BIBO stability and ultimate tracking error bound are rigorously analyzed based on the proposed robust TLC's specific structure. It is shown that the ultimate upper bound of closedloop tracking error monotonously decreases with the product of LESO's and controller's bandwidth. Thus, the analysis provides a guideline to select the two tuning parameters. The theoretical study is further supported by the simulation results. Both stability analysis and simulation results validate the effectiveness of the proposed method.

\section{Conflict of Interests}

The authors declare that there is no conflict of interests regarding the publication of this paper.

\section{Acknowledgments}

This research has been funded in part by the National Natural Science Foundation of China under Grant 61175084/F030601 and in part by Program for Changjiang Scholars and Innovative Research Team in University under Grant IRT 13004.

\section{References}

[1] J. Zhu, A. S. Hodel, K. Funston, and C. E. Hall, "X-33 ascent flight control design by trajectory Linearization-a singular perturbation approach," in Guidance and Control 2001, vol. 107 of Advances in the Astronautical Sciences, pp. 151-170, Univelt, 2001.

[2] J. Zhu, B. D. Banker, and C. E. Hall, "X-33 ascent flight controller design by trajectory linearization-a singular perturbational approach," in Proceedings of the AIAA Guidance, Navigation, and Control Conference, AIAA-2000-4159, Denver, Colo, USA, August 2000.

[3] R. Huang, Y. Liu, and J. J. Zhu, "Guidance, navigation, and control system design for tripropeller vertical-takeoff-andlanding unmanned air vehicle," AIAA Journal of Aircraft, vol. 46, no. 6, pp. 1837-1856, 2009.

[4] B. Zhu and W. Huo, "Adaptive Trajectory Linearization Control for a model-scaled helicopter with uncertain inertial parameters," in Proceedings of the Chinese Control Conference, pp. 43894395, Hefei, China, 2012.

[5] T. M. Adami and J. J. Zhu, "6DOF flight control of fixedwing aircraft by trajectory linearization," in Proceedings of the American Control Conference (ACC '11), pp. 1610-1617, San Francisco, Calif, USA, July 2011.
[6] Y. Liu and J. J. Zhu, "Regular perturbation analysis for trajectory linearization control," in Proceedings of the American Control Conference (ACC '07), pp. 3053-3058, New York, NY, USA, July 2007.

[7] Y. Liu, R. Huang, and J. Zhu, "Adaptive neural network control based on trajectory linearization control," in Proceedings of the 6th World Congress on Intelligent Control and Automation (WCICA '06), pp. 417-421, Dalian, China, June 2006.

[8] X. Li, Y. Li, L. I. Xia, J. Peng, and C. Li, "Robust trajectory linearization control design for unmanned aerial vehicle path following," Journal of Systems Engineering and Electronics, vol. 34, no. 6, pp. 767-772, 2012.

[9] L. Zhu, C. Jiang, H. Chen, and W. Fang, "Direct adaptive trajectory linearization control of aerospace vehicle using SHLNN," Journal of Astronautics, vol. 27, no. 3, pp. 338-353, 2006.

[10] L. Zhu, C. Jiang, and Y. Xue, "Robust adaptive trajectory linearization control for aerospace vehicle using single hidden layer neural networks," Journal of China Ordnance, vol. 29, no. 1, pp. 52-56, 2008.

[11] X. Yali and J. Changsheng, "Trajectory linearization control of an aerospace vehicle based on RBF neural network," Journal of Systems Engineering and Electronics, vol. 19, no. 4, pp. 799-805, 2008.

[12] Y. Yang, "Direct robust adaptive fuzzy control (DRAFC) for uncertain nonlinear systems using small gain theorem," Fuzzy Sets and Systems, vol. 151, no. 1, pp. 79-97, 2005.

[13] J. Changsheng, Z. Chunyu, and Z. Liang, "Research of robust adaptive trajectory linearization control based on T-S fuzzy system," Journal of Systems Engineering and Electronics, vol. 19, no. 3, pp. 537-545, 2008.

[14] R. Huang and J. J. Zhu, “Time-varying high-gain trajectory linearization observer design," in Proceedings of the American Control Conference (ACC '09), pp. 4628-4635, St.Louis, Mo, USA, June 2009.

[15] E. Kim, "A fuzzy disturbance observer and its application to control," IEEE Transactions on Fuzzy Systems, vol. 10, no. 1, pp. 77-84, 2002.

[16] E. Kim, "A discrete-time fuzzy disturbance observer and its application to control," IEEE Transactions on Fuzzy Systems, vol. 11, no. 3, pp. 399-410, 2003.

[17] A. Radke and Z. Gao, "A survey of state and disturbance observers for practitioners," in Proceedings of the American Control Conference (ACC '06), pp. 5183-5188, June 2006.

[18] R. Kotina, Z. Gao, and A. J. van den Bogert, "Modeling and control of human postural sway," in Proceedings of the 20th Congress of the International Society of Biomechanics, July 2005.

[19] R. Miklosovic and Z. Gao, "A dynamic decoupling method for controlling high performance turbofan engines," in Proceedings of the 16th Triennial World Congress of International Federation of Automatic Control (IFAC '05), pp. 532-537, Prague, Czech Republic, July 2005.

[20] Z. Gao, "On the centrality of disturbance rejection in automatic control," ISA Transactions, 2014.

[21] Q. Zheng, L. Dong, D. H. Lee, and Z. Gao, "Active disturbance rejection control for MEMS gyroscopes," IEEE Transactions on Control Systems Technology, vol. 17, no. 6, pp. 1432-1438, 2009.

[22] B. Guo and Z. Zhao, "On the convergence of an extended state observer for nonlinear systems with uncertainty," Systems and Control Letters, vol. 60, no. 6, pp. 420-430, 2011. 


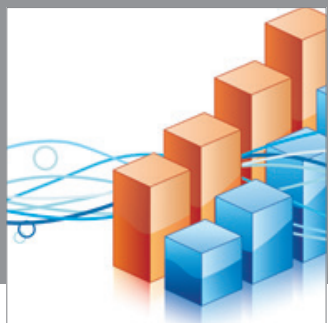

Advances in

Operations Research

mansans

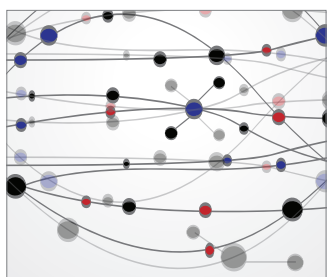

The Scientific World Journal
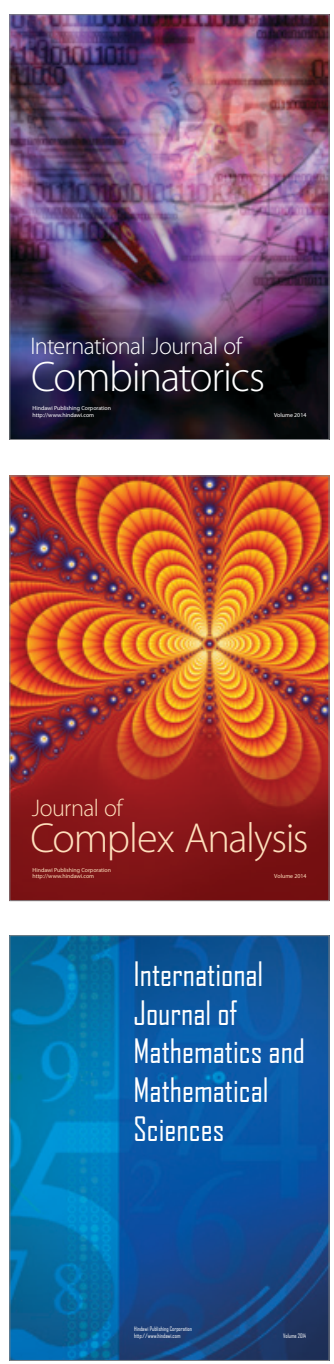
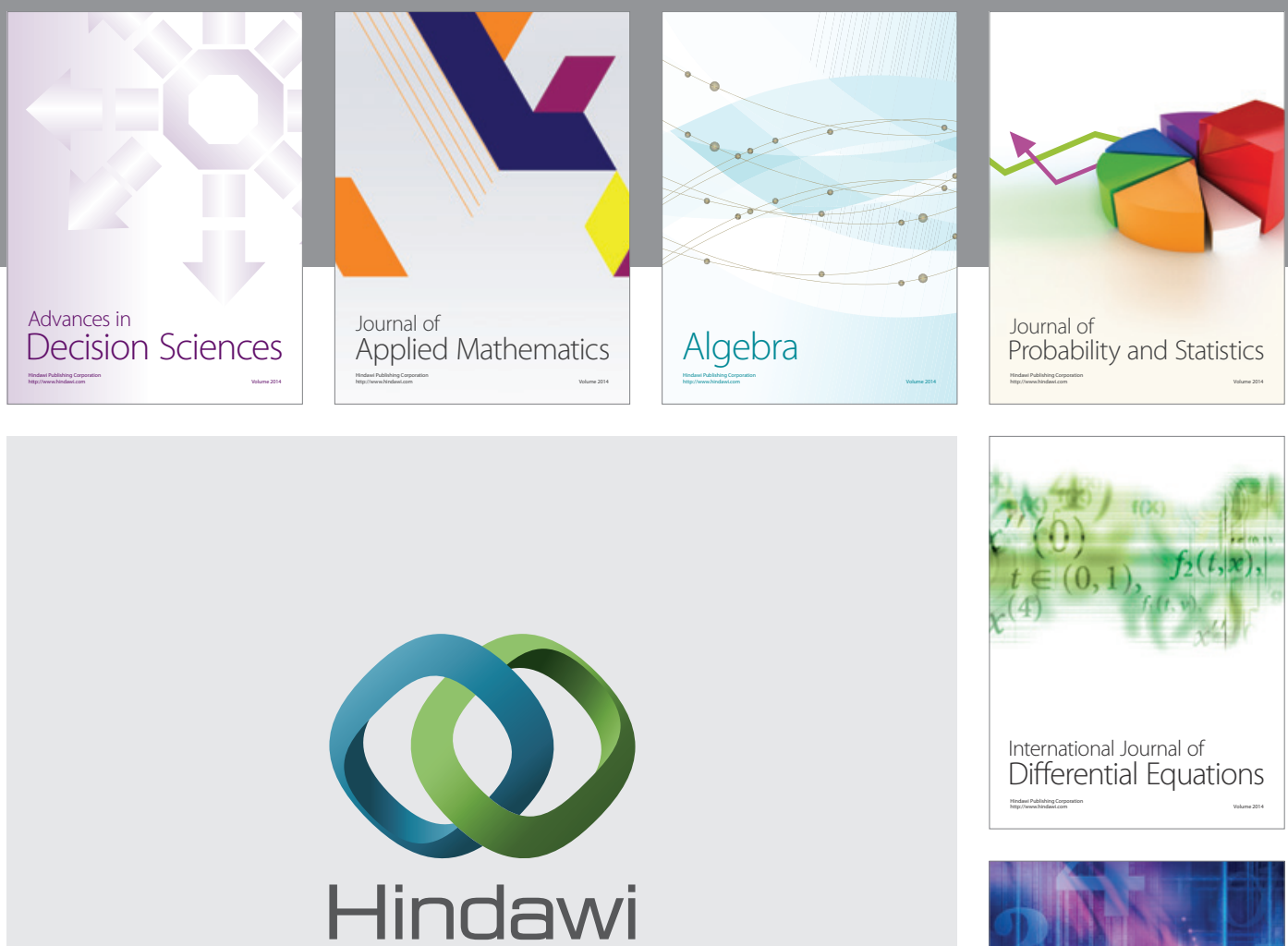

Submit your manuscripts at http://www.hindawi.com
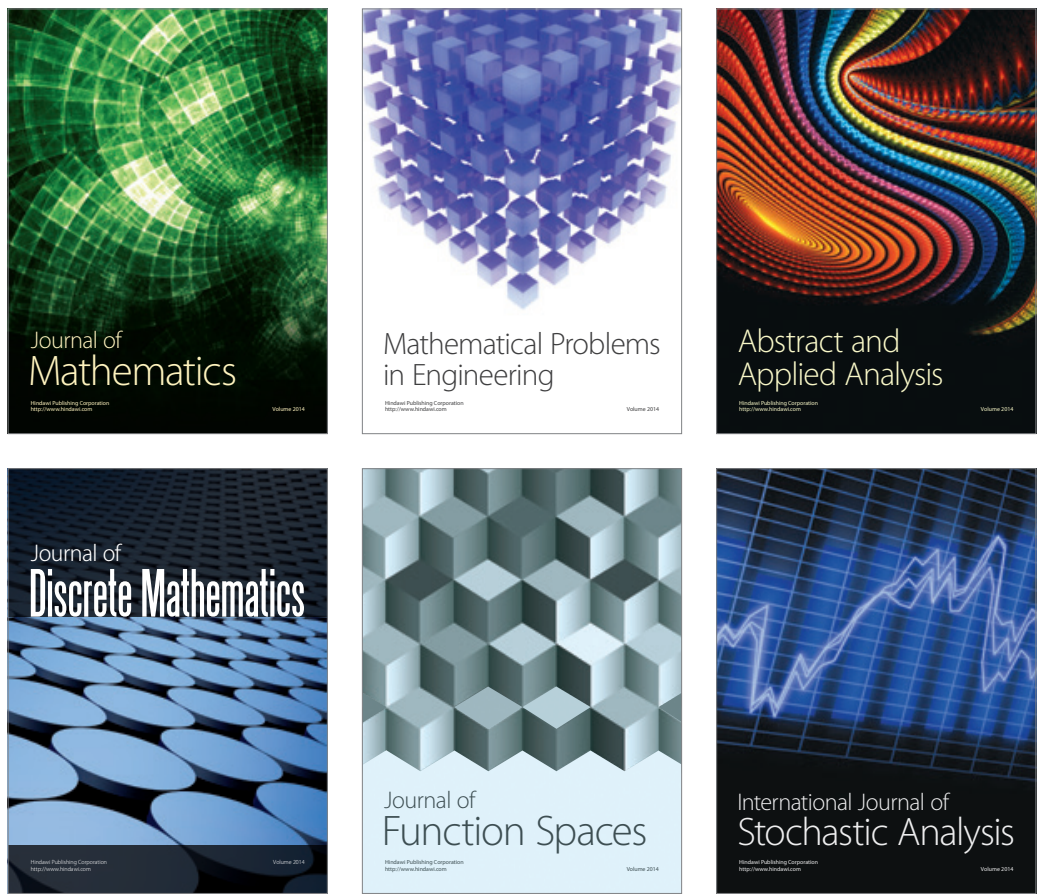

Journal of

Function Spaces

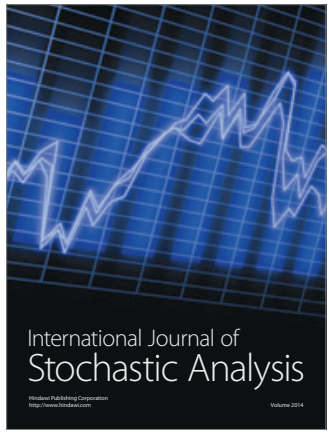

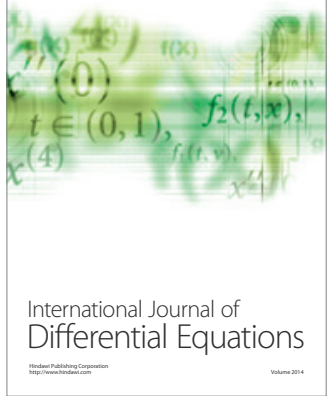
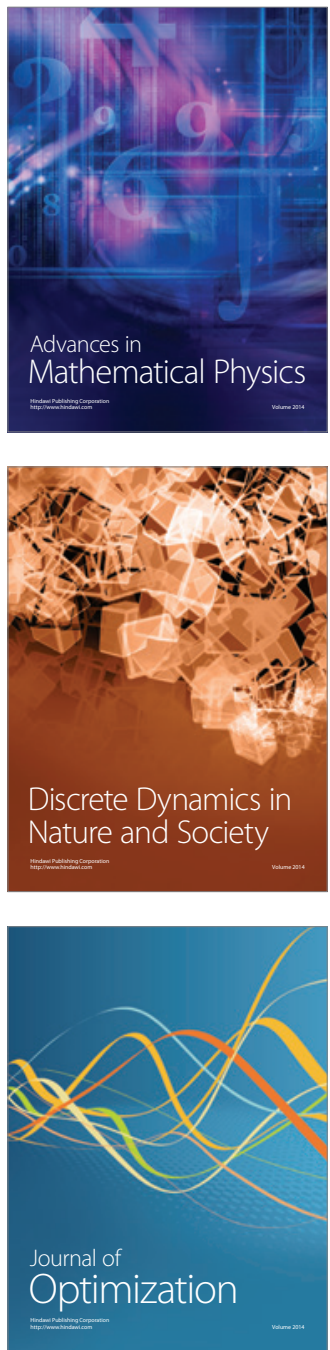